\title{
Generalized Belief Propagation Based TDMR Detector and Decoder
}

\author{
Chaitanya Kumar Matcha*, Mohsen Bahrami ${ }^{\dagger}$, Shounak Roy*, Shayan Garani Srinivasa*, and Bane Vasić ${ }^{\dagger}$ \\ *Department of Electronic Systems Engineering, Indian Institute of Science, Bengaluru, 560012 India. \\ \{mchaitanyakumar, shounak, shayan.gs\}@dese.iisc.ernet.in \\ ${ }^{\dagger}$ Department of Electrical and Computer Engineering, University of Arizona, Tucson, AZ 85721 USA. \\ \{bahrami, vasic\}@ece.arizona.edu
}

\begin{abstract}
Two dimensional magnetic recording (TDMR) achieves high areal densities by reducing the size of a bit comparable to the size of the magnetic grains resulting in two dimensional (2D) inter symbol interference (ISI) and very high media noise. Therefore, it is critical to handle the media noise along with the 2D ISI detection. In this paper, we tune the generalized belief propagation (GBP) algorithm to handle the media noise seen in TDMR. We also provide an intuition into the nature of hard decisions provided by the GBP algorithm. The performance of the GBP algorithm is evaluated over a Voronoi based TDMR channel model where the soft outputs from the GBP algorithm are used by a belief propagation (BP) algorithm to decode low-density parity check (LDPC) codes.
\end{abstract}

\section{INTRODUCTION}

Two dimensional magnetic recording (TDMR) is a promising technology to increase the areal densities beyond 800 $\mathrm{Gb} / \mathrm{in}^{2}$ using sophisticated signal processing algorithms on the currently available magnetic medium by reducing the track width. The signal processing algorithms in TDMR have to handle the 2D ISI and very high media noise arising due to irregularities in the medium.

The correlation and data dependent nature of the media noise can be used to reduce the effect of media noise on the signal processing algorithms in TDMR. Khatami and Vasić [1] have used constrained codes along with GBP detector to avoid harmful patterns that contribute to high media noise. Matcha and Srinivasa [2] have used pattern dependent noise prediction filters along with a 2D soft-output Viterbi algorithm (2D SOVA) to handle the media noise.

We use generalized belief propagation (GBP) algorithm for signal detection. The GBP algorithm is a graph based iterative algorithm where the messages are passed across regions instead of between nodes as seen in the belief propagation (BP) algorithm [3]. The performance of the GBP algorithm in relation to the MAP/ML criteria and the optimal choice of regions is not well understood.

In this paper, we model the media noise from a Voronoi based media model as a pattern dependent noise. We formulate the GBP algorithm to handle the media noise and obtain soft-outputs useful to decode a LDPC code. We also provide

The work at Indian Institute of Science, Bangalore is funded by the Department of Electronics and Information Technology (DeitY), India (gran no. MITO0101). The work at University of Arizona, Tuscon, USA was supported by the National Science Foundation under Grant CCF-1314147 and ECCS-1500170 and in part by IDEMA-ASTC. intuition into the nature of hard decisions given by GBP by looking at the GBP as a convex optimization problem.

The paper is organized as follows: In Section II, we describe the Voronoi based TDMR channel model. In Section III, we provide insights into the nature of hard decisions from GBP algorithm and formulate the algorithm to handle media noise. In Section IV, we use GBP algorithm to bound the TDMR channel capacity for designing the LDPC code of appropriate rate. We also discuss the numerical results where LDPC codes are decoded using soft outputs from the GBP algorithm.

\section{TDMR CHANNEL MODEL}

A TDMR channel model is characterized by a magnetic media model, a write process and a read-back procedure. For the study in this paper, we use a Voronoi based channel model proposed in [1]. This section provides the essential details of the channel model.

\section{A. Voronoi Based Magnetic Medium}

The non-ideal features of the magnetic medium are modeled as Voronoi tiling of magnetic grains. The distribution of grain centers is modeled as the Poisson disk process with boundary sampling as explained in [1]. The distribution is characterized by the center-to-center (CTC) distance parameter which defines the minimum distance between any two grains.

For the storage of information, the magnetic medium is viewed as a rectangular grid. Each rectangular cell has a size of bit period $(\mathrm{BP}) \times$ track width $(\mathrm{TW})$ and stores a channel bit. The write head magnetizes all grains whose centers are within a bit area with a polarization corresponding to the channel bit.

\section{B. Read Procedure and Media Noise}

The read-head output samples are obtained by convolving the magnetization of recorded medium with the read-head response and then sampling at the centers of bit cells. We consider the read-head impulse response to be a Gaussian pulse truncated to $3 \times 3$ bit area [1]. We denote $P W_{50}^{C T}$ $\left(P W_{50}^{D T}\right)$ as the width of Gaussian pulse at half-amplitude in the down-track (cross-track) direction.

The media noise arises due to the grains near the boundaries of the bit region and is correlated in both down-track and cross-track directions. If two neighboring bits have the same polarization, there is no media noise caused from that border. For the $3 \times 3$ span of read-head impulse response, the media 
noise is dependent on the local $3 \times 3$ data patterns written on the medium. We can approximate the pattern dependent media noise distribution as a Gaussian distribution [4].

For our discussion, let $x_{i, j} \in\{0,1\},(i, j) \in \mathbb{Z}^{2}$ represent bits written onto the medium. Let $y_{i, j} \in \mathbb{R},(i, j) \in \mathbb{Z}^{2}$ represent the read-back samples obtained from the channel model. Let $\mathbf{x}=\left[x_{i, j}\right] \in\{0,1\}^{n \times m}$ represent a $n \times m$ page of bits and $\mathbf{y}=\left[x_{i, j}\right] \in \mathbb{R}^{n \times m}$ represent the corresponding page of read-back samples obtained from the channel model. Let $\mathbf{x}_{i, j}$ represent the set of bits that contribute to the read-back sample $y_{i, j}$. For a channel with $3 \times 3$ ISI span, $\mathbf{x}_{i, j}$ contains 9 bits $\left\{x_{i+k, j+l} \mid k, l \in\{-1,0,1\}\right\}$.

\section{GBP BASED 2D-ISI DETECTION}

Generalized belief propagation (GBP) algorithm is a graph based decoding/detection algorithm that can be formulated as a convex optimization problem that minimizes the Gibbs free energy [3]. The algorithm provides a method to approximate marginal distributions which makes it suitable for MAP detection with soft outputs.

The GBP algorithm is known to give exact marginals if and only if the region based graph has no loops [5]. Even though the region based graphs always contain loops when used for 2D ISI signal detection, the GBP algorithm provides a method to approximate the marginals that are empirically observed to be close to the actual marginals.

In this section, we provide insights into the nature of hard decisions from the GBP algorithm and evaluate the performance of the GBP algorithm over a chosen 2D ISI channel for different choices of region. We next formulate the GBP algorithm for the noise characteristics seen in the Voronoi based TDMR channel model in Section II.

\section{A. Gibbs free energy and Kikuchi approximation}

Assuming uniform distribution of the input bits and white noise samples in the channel model, the a-posteriori probability of $\mathbf{x}$ given read-back samples $\mathbf{y}$ is given by

$$
\begin{aligned}
& p(\mathbf{x} \mid \mathbf{y})=p(\mathbf{y} \mid \mathbf{x}) p(\mathbf{x}) p(\mathbf{y})^{-1} \propto p(\mathbf{y} \mid \mathbf{x}) \\
& p(\mathbf{y} \mid \mathbf{x})=\prod_{i, j} f_{i, j}\left(\mathbf{x}_{i, j}\right)
\end{aligned}
$$

where $f_{i, j}\left(\mathbf{x}_{i, j}\right)=p\left(y_{i, j} \mid \mathbf{x}_{i, j}\right)$ is the distribution function of noise sample at location $(i, j)$. Therefore, we have

$$
p(\mathbf{x} \mid \mathbf{y})=\frac{1}{Z} \prod_{i, j} f_{i, j}\left(\mathbf{x}_{i, j}\right),
$$

for some $Z(\mathbf{y})$. Let $b(\mathbf{x})$ represent the belief of the a-posterior probability (APP). From the properties of KL-divergence, the belief $b(\mathbf{x})=p(\mathbf{x} \mid \mathbf{y})$ can be achieved by minimizing the free energy given by

$$
\begin{aligned}
F=E-H & =\mathcal{D}(b(\mathbf{x}) \| p(\mathbf{x} \mid \mathbf{y}))-\ln Z(\mathbf{y}), \\
\text { average energy } E & =-\sum_{i, j} \sum_{\mathbf{x}_{i, j}} b\left(\mathbf{x}_{i, j}\right) \ln f_{i, j}\left(\mathbf{x}_{i, j}\right), \\
\text { entropy } H & =\sum_{\mathbf{x}} b(\mathbf{x}) \ln b(\mathbf{x}) .
\end{aligned}
$$

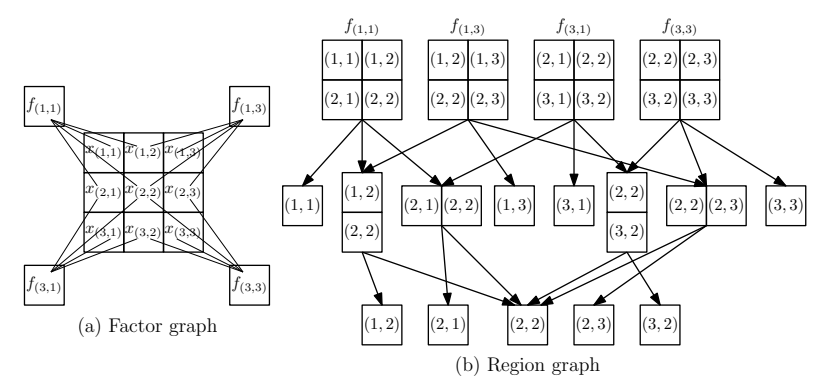

Figure 1. Factors $f_{i, j}(\cdot)$ of a $3 \times 3$ page are shown. The corresponding region graph with all regions and sub-regions is also shown. The arrows in the region graph show the flow of messages in the GBP algorithm.

Let a region $R \subset \mathbb{R}^{2}$ be defined as a set of positions within a page. Let $\mathcal{R}$ represent a collection of such regions such that each of $\mathbf{x}_{i, j}$ is included in atleast one region. For each $R \in \mathcal{R}$, let $\mathbf{x}_{R}$ be the vector of bits in the region $R$ and $b\left(\mathbf{x}_{R}\right)$ and $p\left(\mathbf{x}_{R}\right)$ be the corresponding marginal beliefs and probabilities. The regions are partially ordered based on the containment of one region inside another [3]. A region graph is formed using this partial ordering as shown in Figure 1.

The free energy is approximated using the entropy of individual regions as

$$
\begin{aligned}
\hat{F}= & -\sum_{i, j} \sum_{\mathbf{x}_{i, j}} b\left(\mathbf{x}_{i, j}\right) \ln f_{i, j}\left(\mathbf{x}_{i, j}\right) \\
& +\sum_{R \in \mathcal{R}} c_{R} \sum_{\mathbf{x}_{R}} b\left(\mathbf{x}_{R}\right) \ln b\left(\mathbf{x}_{R}\right),
\end{aligned}
$$

where $c_{R}$ are overcounting numbers defined as $c_{R}=$ $\sum_{p \in \mathcal{P}_{R}} 1-c_{p}$ and $\mathcal{P}_{R}$ are parents of region $R$ in the region graph. This approximation is called Kikuchi approximation or region based approximation (RBA). The marginals $b\left(\mathbf{x}_{R}\right)$ are estimated by minimizing (6) under the constraints

$$
\sum_{u \in \mathbf{x}_{p \backslash R}} b\left(\mathbf{x}_{p}\right)=b\left(\mathbf{x}_{R}\right) \quad \forall p \in \mathcal{P}_{R}, \forall R \in \mathcal{R} .
$$

These constraints ensure that the beliefs of sub-regions are obtained by marginalizing the beliefs of their parents [5]. The message update rules of GBP algorithm are obtained from the constrained optimization of $\hat{F}$ using Lagrange multipliers.

The regions and $c_{R}$ are chosen to 1 ) ensure unique solution to the for GBP algorithm, 2) closely approximate the marginals 3) reduce computational complexity.

\section{B. Hard Decisions from GBP}

The analysis on GBP in the literature is focused on closely approximating the marginals (soft decisions). However, the nature of hard decisions is also of interest while analyzing GBP as a signal detection/decoding algorithm. In this subsection, we use the ideas of linear programming and convex optimization to provide an insight into the behavior of GBP algorithm for hard decisions decoding/detection.

Hard decision decoding is a signal classification problem where the received signal is classified based on the decision regions with in a signal space. We define the spaces of interest and the corresponding decision regions as follows. 
Let $\{0,1\}^{N}=\left\{\mathbf{m}_{0}, \mathbf{m}_{1}, \cdots, \mathbf{m}_{2^{N}-1}\right\}$ represent the set of states taken by $\mathbf{x}$, where $N=m n$ is the number of bits in a page, $m_{k}(i, j)$ is the value of $x_{i, j}$ when $\mathbf{x}=\mathbf{m}_{k}$. Let $\mathbf{m}_{i}(R)$ be the vector of bits in $\mathbf{m}_{i}$ restricted to the region $R \in \mathcal{R}$.

The RBA reduces the optimization problem in $b(\mathbf{x})$ space to the optimization in a lower dimensional space of marginals $\left\{b\left(\mathbf{x}_{R}\right)\right\}_{R \in \mathcal{R}}$. Let $\mathbf{b}$ be the vector of beliefs $b(\mathbf{x}), \mathbf{x}=$ $\mathbf{m}_{0} \cdots \mathbf{m}_{2^{N}-1}$, and let $\mathbf{b}_{\mathcal{R}}$ represent the vector of marginals $b\left(\mathbf{x}_{R}\right), \mathbf{x}_{R}=\mathbf{m}_{0}(R) \cdots \mathbf{m}_{2^{N-1}}(R), R \in \mathcal{R}$. We define the space of probabilities and marginals as follows.

Definition 1. Probability space: We define $\Delta$ as the space of probabilities/beliefs $\mathbf{b}(\mathbf{x})$ with the constraints

$$
0 \leq b\left(\mathbf{x}=\mathbf{m}_{i}\right) \leq 1 \text { and } \sum_{i=0}^{2^{N}-1} b\left(\mathbf{x}=\mathbf{m}_{i}\right)=1 .
$$

Let $\left\{\mathbf{v}_{0}, \mathbf{v}_{1}, \cdots, \mathbf{v}_{2^{N}-1}\right\}$ represent the vertices of the space where $\mathbf{v}_{i}$ represents $b\left(\mathbf{x}=\mathbf{m}_{i}\right)=1, i=0, \cdots, 2^{N}-1$. Let $\mathbf{u}$ be the uniform distribution.

Marginal space: Let $\Delta_{M}$ be the space of marginals $\mathbf{b}_{\mathcal{R}}(\mathbf{x})$ for the regions in $\mathcal{R}$ with the constraints $0 \leq b\left(\mathbf{x}_{R}\right) \leq 1$ and $\sum_{\mathbf{x}_{R}} b\left(\mathbf{x}_{R}\right)=1 \forall R \in \mathcal{R}$. We further enforce following constraints such that the marginals of two overlapping regions are consistent:

$$
\sum_{\mathbf{x}_{R_{i} \backslash R_{j}}} b\left(\mathbf{x}_{R_{i}}\right)=\sum_{\mathbf{x}_{R_{j} \backslash R_{i}}} b\left(\mathbf{x}_{R_{j}}\right) \quad \forall R_{i}, R_{j} \in \mathcal{R} .
$$

Remark: We can define a linear map $\mathcal{L}: \Delta \rightarrow \Delta_{M}$ using the marginalization operations $b\left(\mathbf{x}_{R}\right)=\sum_{\mathbf{x}_{R^{c}}} b(\mathbf{x}), R \in \mathcal{R}$.

Let $\hat{\mathbf{v}}_{i}=\mathcal{L}\left(\mathbf{v}_{i}\right), i=0, \cdots, 2^{N}-1$ and $\hat{\mathbf{u}}=\mathcal{L}(\mathbf{u})$.

Definition 2. Pseudo-marginal space: Let $\Delta_{P}$ be a space of pseudo-marginals $\mathbf{b}_{\mathcal{R}}(\mathbf{x})$ for regions in $\mathcal{R}$ with the constraints $0 \leq b\left(\mathbf{x}_{R}\right) \leq 1$ and $\sum_{\mathbf{x}_{R}} b\left(\mathbf{x}_{R}\right)=1 \forall R \in \mathcal{R}$. These are pseudo marginals as we ignored the constraints in (8). Therefore, $\Delta_{M} \subset \Delta_{P}$.

Definition 3. Optimal hard decisions: The word $\mathbf{m}_{F E R}$ is said to be frame error rate (FER) optimal hard decision if

$$
p\left(\mathbf{x}=\mathbf{m}_{F E R}\right)>p\left(\mathbf{x}=\mathbf{m}_{j}\right), \quad \forall \mathbf{m}_{j} \neq \mathbf{m}_{F E R} .
$$

The word $\mathbf{m}_{B E R}$ is bit error rate (BER) optimal decision if

$$
p\left(x_{i, j}=\mathbf{m}_{B E R}(i, j)\right)>0.5, \quad \forall x_{i, j} .
$$

Let $\mathbf{v}_{F E R}, \mathbf{v}_{B E R}$ ( and $\hat{\mathbf{v}}_{F E R}, \hat{\mathbf{v}}_{B E R}$ ) be the vertices in $\Delta($ and $\left.\Delta_{M}\right)$ corresponding to $b\left(\mathbf{m}_{F E R}\right)=1$ and $b\left(\mathbf{m}_{B E R}\right)=$ 1. Since the inner product $\left\langle\mathbf{v}_{i}, \mathbf{b}\right\rangle=b\left(\mathbf{x}=\mathbf{m}_{i}\right)$, the FER decision region can be written using (9) as

$$
D_{(F E R)}=\Delta \cap \bigcap_{j: \mathbf{m}_{j} \neq \mathbf{m}_{F E R}}\left\{\left\langle\mathbf{v}_{F E R}-\mathbf{v}_{j}, \mathbf{b}\right\rangle \geq 0\right\}
$$

It is easy to see that all FER decision regions corresponding to each word $\mathbf{m}_{i}$ intersect at $\mathbf{u}$. Proposition 1 identifies the FER decision region in $\Delta_{P}$ corresponding to (11).
Proposition 1. The optimal FER decision region in the pseudo marginal space $\Delta_{P}$ is

$$
\hat{D}_{F E R}=\Delta_{P} \cap \bigcap_{j: \hat{\mathbf{v}}_{j} \neq \hat{\mathbf{v}}_{F E R}}\left\{\left\langle\hat{\mathbf{v}}_{F E R}-\hat{\mathbf{v}}_{j}, \mathbf{b}_{\mathcal{R}}\right\rangle \geq 0\right\} .
$$

Proof: Let $\tilde{D}_{F E R}=\mathcal{L}\left(D_{F E R}\right)$ be the linear map of $D_{F E R}$ from $\Delta$ to $\Delta_{M}$. Note that $\tilde{D}_{F E R}$ also has linear decision boundaries and hence the decision boundaries in $\Delta_{P}$ are also linear. Notice that $D_{F E R}$ and $\hat{D}_{F E R}$ are Voronoi regions in their own spaces.

Each vertex of $D_{F E R}$ is obtained as follows: Choose any subset set of points $\mathcal{V} \subseteq\left\{\mathbf{v}_{i} \mid i=1, \cdots, 2^{N-1}\right\}$. Centroid of points $\mathcal{V} \cup\left\{\mathbf{v}_{F E R}\right\}$ is a vertex of $D_{F E R}$.

Similarly, the vertices of $\hat{D}_{F E R}$ in (12) are the centroids of a subset of points from $\left\{\hat{\mathbf{v}}_{i} \neq \hat{\mathbf{v}}_{F E R}\right\}$ and $\hat{\mathbf{v}}_{F E R}$. Since the map from $\mathbf{v}_{i}$ to $\hat{\mathbf{v}}_{i}$ is linear, the same linear map maps the centriods in $\Delta$ to centriods in $\Delta_{P}$. Therefore, the vertices of $\hat{D}_{F E R}$ in (12) are a map of vertices of $D_{F E R}$. Therefore, $\hat{D}_{F E R}$ in (12) is the optimal FER decision region in $\Delta_{P}$.

The following proposition proves a property of the average energy in $\Delta_{P}$ that helps us in understanding the nature of signal classification by GBP.

Proposition 2. In the pseudo marginal space $\Delta_{P}$, the average energy term in the Kikuchi approximation of free energy has a constant gradient $\mathbf{g}=\frac{\partial E}{\partial \mathbf{b}_{\mathcal{R}}}$ satisfying

$$
\left\langle\hat{\mathbf{v}}_{F E R}-\hat{\mathbf{v}}_{i}, \mathbf{b}_{\mathcal{R}}\right\rangle \leq 0 \forall \hat{\mathbf{v}}_{i} \neq \hat{\mathbf{v}}_{F E R}, i=0, \cdots, 2^{N}-1 .
$$

Proof: The gradient of $E$ has the terms $-\log f_{i, j}\left(\mathbf{x}_{i, j}\right)$ and hence is constant. Since $E=0$ when $\mathbf{b}_{\mathcal{R}}=\mathbf{0}$, we can write $E\left(\mathbf{b}_{\mathcal{R}}\right)=\left\langle\mathbf{b}_{\mathcal{R}}, \mathbf{g}\right\rangle$. From (4), $E$ is linear in $\Delta$ and the minima of $E$ occurs on the boundaries of $\Delta$. We can easily verify that $E$ is minimized in $\Delta$ when $b\left(\mathbf{x}=\mathbf{m}_{F E R}\right)=1$ i.e., at the point $\mathbf{b}=\mathbf{v}_{F E R}$. Consider the polytope in $\Delta_{P}$ formed by the points $\hat{\mathbf{v}}_{i}, i=0, \cdots, 2^{N}-1$. Using the exactness of the average energy in RBA [3], we can claim that the average energy is minimum at $\mathbf{b}_{\mathcal{R}}=\hat{\mathbf{v}}_{F E R}$ inside this polytope i.e., $E\left(\hat{\mathbf{v}}_{F E R}\right) \leq E\left(\hat{\mathbf{v}}_{i}\right) \Longrightarrow\left\langle\hat{\mathbf{v}}_{F E R}-\hat{\mathbf{v}}_{i}, \mathbf{b}_{\mathcal{R}}\right\rangle \forall \hat{\mathbf{v}}_{i} \neq \hat{\mathbf{v}}_{F E R}$.

Nature of signal classification by GBP: The approximated entropy $\hat{H}$ has maximum value at uniform distribution $\hat{\mathbf{u}}$. Proposition 2 shows that the gradient of $-E$ has the largest component along the direction of $\hat{\mathbf{v}}_{F E R}$ than in the direction of any other $\hat{\mathbf{v}}_{i}$. Therefore, in the optimization problem to maximize $-E+H$, the component $-E$ shifts the maxima of $\hat{H}$ closer to $\hat{\mathbf{v}}_{F E R}$ i.e., within the region $\hat{D}_{F E R}$. This shows that the inherent nature of signal classification achieved by GBP is towards optimizing FER. Due to this nature of signal classification, the GBP algorithm is suitable for hard decision decoding of error correcting codes (ECC) where FER has to be minimized. A good approximation of entropy will provide a closer approximation of the marginals resulting in optimal BER. Therefore, a good approximation of entropy is the key for the problems where BER has to be minimized.

\section{Choice of regions}

In this subsection, we focus on choosing regions suitable for $3 \times 3$ ISI span. The optimal choice of regions is not trivial. 


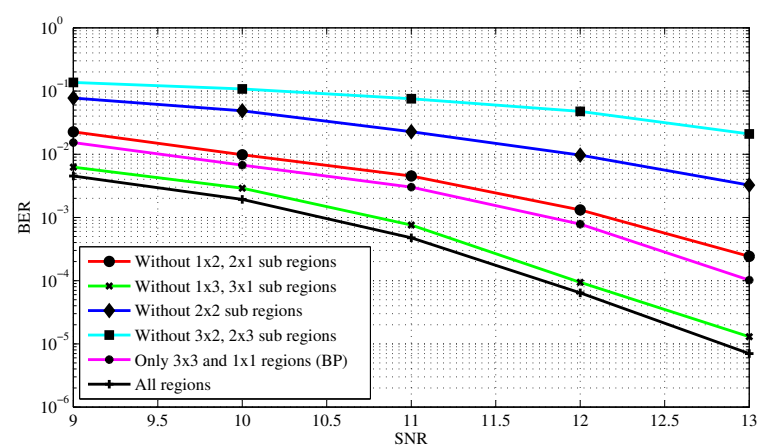

Figure 2. BER performance of the GBP algorithm for different choices of regions. The best performance is seen when all sub-regions of sizes $2 \times 3$, $3 \times 2,1 \times 3,3 \times 1,2 \times 1,1 \times 2,2 \times 2,1 \times 1$ are chosen. Omitting any of the regions would not ensure that the beliefs marginalize to the same values in the intersection of regions. Severe degradation in performance is seen if there are large number of descendants for the omitted regions.

Welling [6] has proposed a region pursuit algorithm based on his observations on splitting and merging of regions. However, the choice of regions larger than $3 \times 3$ is computationally prohibitive for signal detection in TDMR.

Therefore, we restrict our search to regions of size $3 \times 3$ or smaller. Let $\mathcal{R}_{p \times q}$ denote the set of regions of size $p \times q$ within a frame. The valid sizes of sub-regions are $2 \times 3,3 \times 2$, $1 \times 3,3 \times 1,2 \times 1,1 \times 2,2 \times 2,1 \times 1$. Let $\mathcal{R}^{\prime}$ denote the collection of all $3 \times 3$ regions and sub-regions within a frame.

The choice $\mathcal{R}=\mathcal{R}^{\prime}$ is shown in [5] to ensure several desirable properties for the convex optimization problem:

1) The constraints in (7) ensure that the beliefs of regions are marginals of a distribution if and only if $\mathcal{R}=\mathcal{R}^{\prime}$.

2) The choice achieves totally balanced condition that helps in removing bias in the approximation of entropy.

3) The choice ensures unique solution.

Figure 2 shows the performance of the GBP algorithm for different choices of regions over a 2D-ISI AWGN channel given in [7]. The BER is estimated by detecting pages of size $32 \times 32$ at a time. We notice that the best performance is obtained when the $\mathcal{R}=\mathcal{R}^{\prime}$. The performance is about $0.2 \mathrm{~dB}$ better than JTED [7] for the same 2D ISI channel operating on $64 \times 64$ pages. We also notice severe degradation in performance if the omitted set of regions has a large number of descendants.

\section{Soft Information from GBP}

As discussed in Section III-C, the GBP algorithm can be used to compute the a-posteriori probabilities of the bits. In the following, we formulate the problem of extracting soft information from the Voronoi based TDMR channel as an instance of 2D ISI channels.

The APP ratios in the log domain, also called the $\log$ likelihood ratio for each bit $x_{i, j}$ is approximated using the beliefs from GBP algorithm as

$\operatorname{LLR}\left(x_{i, j}\right)=\log \left(\frac{p\left(x_{i, j}=1 \mid \mathbf{y}\right)}{p\left(x_{i, j}=0 \mid \mathbf{y}\right)}\right) \approx \log \left(\frac{b\left(x_{i, j}=1\right)}{b\left(x_{i, j}=0\right)}\right)$.
Table I

$\mathrm{RS}_{\mathrm{CT}}\left(\mathrm{RS}_{\mathrm{DT}}\right)$ DENOTES THE READER RESPONSE SPAN IN CROSS-TRACK (DOWN-TRACK) DIMENSION. ALL THE PARAMETERS IN THE TABLE ARE SPECIFIED IN NANOMETERS. * INDICATES THAT THE PARAMETER IS VARIED IN THE SIMULATIONS. CTC $=7 \mathrm{~nm}$.

\begin{tabular}{ccccccc}
\hline & TW & BP & $R S_{C T}$ & $R S_{D T}$ & $P W_{50}^{C T}$ & $P W_{50}^{D T}$ \\
\hline \hline TDMR & $\star$ & 10 & 28 & 28 & 14 & 14 \\
\hline
\end{tabular}

The MAP detection minimizes the BER by maximizing the APP, $p\left(x_{i, j} \mid \mathbf{y}\right)$, for each $x_{i, j}$ in $\mathbf{x}$.

In order to utilize the GBP algorithm for finding the LLRs, the first step is to identify the local constraint functions $f_{i, j}\left(\mathbf{x}_{i, j}\right)$ given in (2). Since the 2D ISI in our model is limited to a $3 \times 3$ span, the read-back sample $y_{i, j}$ and the corresponding media noise sample depends only on $\mathbf{x}_{i, j}$, the $3 \times 3$ bit region centered at $(i, j)$. Therefore, the local constraint functions can be defined using the pattern dependent noise distribution as $f_{i, j}\left(\mathbf{x}_{i, j}\right)=\mathrm{p}\left(y_{i, j} \mid \mathbf{x}_{i, j}\right)$.

We can incorporate the GBP algorithm to the probabilistic graphical model of this problem that we introduced in [1] in order to obtain the APPs. In order to obtain optimal performance, as seen in Section III-C, we choose the regions to include all $3 \times 3$ regions and all possible intersections of these regions.

\section{USING SOFT-INFORMATION FROM GBP FOR \\ ITERATIVE DECODING OF CODED TDMR CHANNELS}

In our simulations, LDPC coded bits are written on and read from the Voronoi based magnetic medium, resulting in read-back samples. The GBP algorithm is used for signal detection and the LLRs from the GBP algorithm are used for iterative LDPC decoding using the belief propagation (BP) algorithm. We design the LDPC code rate by bounding the channel capacity of our TDMR channel model using the GBP based TDMR SIR estimation algorithm described in our recent work [8]. The TDMR channel model parameters used in the simulations are given in Table I. The GBP algorithm detects a $32 \times 32$ page of data at a time. The read-head response is truncated to restrict the ISI span to $3 \times 3$ bit area.

In order to demonstrate the feasibility of implementation of our proposed GBP based TDMR detector, we conducted experiments to recover the user bits from the distorted coded TDMR channel.

\section{A. Lower and Upper Bounds on the SIR of Voronoi Channel}

The SIR between the input and output random processes $X$ and $Y$ of a Voronoi channel is defined as the mutual information per symbol between $X$ and $Y$ when the input distribution is uniform. For a $n \times m$ Voronoi channel, we have $S I R=\frac{1}{n m} I(X ; Y)$ when the input distribution is uniform where $I(X ; Y)=H(Y)-H(Y \mid X)$. The term, $H(Y \mid X)$, is the conditional entropy of the media noise that can be computed analytically using the channel model. As discussed in Subsection II-B, we estimate the media noise distribution $p\left(Y \mid \mathbf{x}_{R}\right)$ by an AWGN with the noise variance $\sigma_{\mathbf{x}_{R}}^{2}$ dependent on each $3 \times 3$ span of input data. Therefore, 


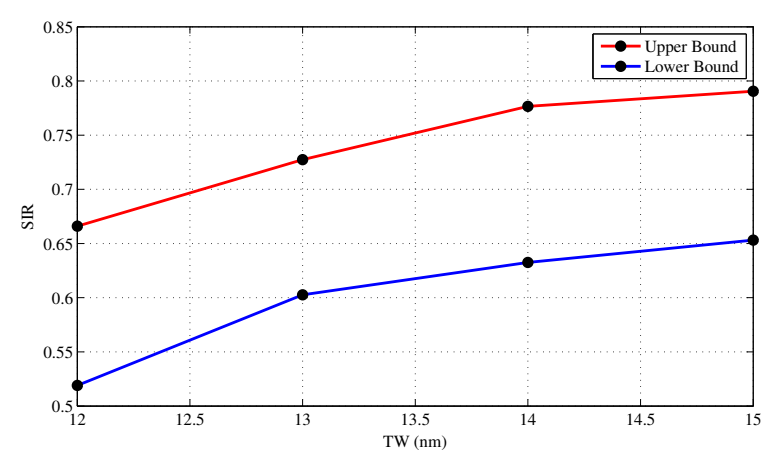

Figure 3. Lower and upper bounds on the SIR of Voronoi channel of TDMR system with the parameters given in Table I

$H\left(Y \mid X=\mathbf{x}_{R}\right)=\frac{1}{2} \log \left(2 \pi e \sigma_{\mathbf{x}_{R}}^{2}\right) . H(Y)$ is obtained using the GBP based TDMR SIR estimation algorithm [8].

The GBP-based capacity estimation algorithm provides a lower bound on the 2D partition function of a factor graph, and accordingly the SIR which is obtained using the algorithm is only an estimate. In [9], GBP was used to estimate the capacity of 2D RLL codes and it was shown that GBP capacity estimate for local constraints are accurate (up to $3^{\text {rd }}$ decimal place). Moreover, in [10], it was shown that SIR, computed for the 2D Gaussian channels using the GBP-based algorithm coincides with the lower and upper bounds of the SIR given by Chen and Siegel [11]. In our recent work [8], we have shown that the lower and upper bounds merge to the SIR of the Voronoi channel by increasing the dimensions of the medium. The upper and lower bounds on SIR are obtained as:

Lower Bound: No information about outside of boundaries of the $32 \times 32$ page is available for the GBP based TDMR SIR estimator. In this case, we compute the beliefs assuming that all states of the boundary regions are equi-probable. This gives us a lower bound on the SIR of the TDMR channel.

Upper Bound: The boundary information of the magnetic medium is assumed to be known to the SIR estimator. In this case, the bit values outside the page boundary are known and treated as deterministic giving us an upper bound on the SIR.

Figure 3 shows the SIR lower and upper bounds for the chosen TDMR channel model. We use SIR as a lower bound on the capacity of the system. Based on the observed lower and upper bounds of the SIR we choose LDPC code rate to be $R=0.66$. The LDPC code of length $N=756$, rate $R=0.66$, and a circulant size of $L=126$ is constructed by methods described in [12], and is free of small trapping sets.

\section{B. Frame Error Rate Results}

Figure 4 shows the frame error rate (FER) results with respect to $T W$ for the Voronoi channel with the parameters given in Table I. At $T W=16.2 \mathrm{~nm}$, the LDPC code gives more than two orders of magnitude gain in the FER when compared with the $\mathrm{BCH}$ code of length 1023 bits, rate 0.66 .

\section{CONCLUSION}

In this paper, we have proposed a method to handle the media noise seen in the TDMR channels using the GBP based

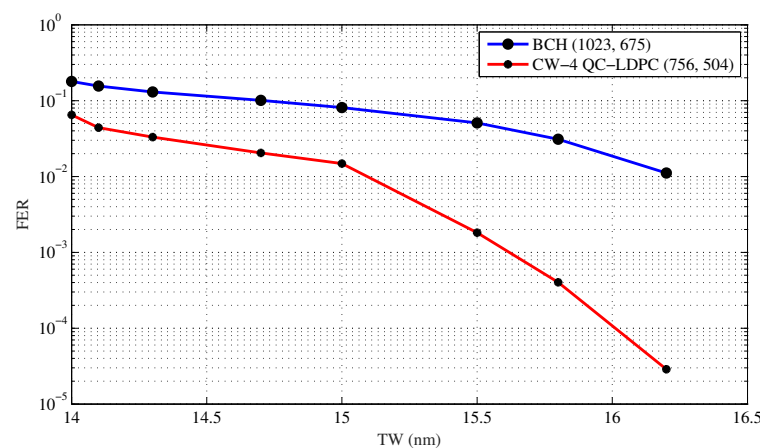

Figure 4. The FER result of quasi-cyclic column weight four LDPC code with $N=756, R=0.66$ with respect to the parameter $T W$ for the Voronoi channel. Also, the FER of BCH code $(1023,675)$ is plotted for reference.

detector. We have used the GBP algorithm for signal detection in conjunction with a BP algorithm for LDPC decoding. We have given an insight into the nature of signal classification (hard decisions) by GBP to be motivated towards minimizing FER. We have also evaluated the performance of the GBP algorithm for different choices of regions suitable for TDMR. The GBP algorithm can be formulated to handle correlation in the media noise and exchange information in a turbo fashion with the BP algorithm for further gains in the TDMR performance.

\section{REFERENCES}

[1] M. Khatami and B. Vasić, "Constrained coding and detection for TDMR using generalized belief propagation," in ICC, Jun. 2014, pp. 3889-3895

[2] C. Matcha and S. G. Srinivasa, "Generalized partial response equalization and data-dependent noise predictive signal detection over media models for TDMR," IEEE Trans. Magn, vol. 51, no. 10, pp. 1-15, Oct 2015.

[3] J. Yedidia, W. Freeman, and Y. Weiss, "Constructing free-energy approximations and generalized belief propagation algorithms," IEEE Trans. Inf. Theory, vol. 51, no. 7, pp. 2282 - 2312, Jul. 2005

[4] M. Khatami, M. Bahrami, and B. Vasić, "Information rates of constrained TDMR channels using generalized belief propagation," in GLOBECOM, San Diego, CA, Dec. 2015, pp. 1-6.

[5] P. Pakzad and V. Anantharam, "Estimation and marginalization using Kikuchi approximation methods," Neural Computation, vol. 17, pp. 1836-1873, 2003.

[6] M. Welling, "On the choice of regions for generalized belief propagation,' in UAI '04. Arlington, Virginia, United States: AUAI Press, 2004, pp. 585-592.

[7] Y. Chen and S. G. Srinivasa, "Joint self-iterating equalization and detection for two-dimensional intersymbol-interference channels," IEEE Trans. Comm, vol. 61, no. 8, pp. 3219-3230, Aug. 2013.

[8] M. Khatami, , M. Bahrami, and B. Vasić, "Symmetric information rate estimation and bit aspect ratio optimization for TDMR using generalized belief propagation," in IEEE Int. Symp. Inf. Theory, Jun. 2015.

[9] G. Sabato and M. Molkaraie, "Generalized belief propagation for the noiseless capacity and information rates of run-length limited constraints," IEEE Trans. Commun., vol. 60, no. 3, pp. 669-675, Mar. 2012

[10] O. Shental, N. Shental, S. Shamai, I. Kanter, A. Weiss, and Y. Weiss, "Discrete-input two-dimensional Gaussian channels with memory: Estimation and information rates via graphical models and statistical mechanics," IEEE Trans. Inf. Theory, vol. 54, no. 4, pp. 1500-1513, Apr. 2008 .

[11] J. Chen and P. Siegel, "On the symmetric information rate of twodimensional finite-state ISI channels," IEEE Trans. Inf. Theory, vol. 52, no. 1, pp. 227-236, Jan 2006.

[12] D. V. Nguyen, S. K. Chilappagari, B. Vasić, and M. W. Marcellin, "On the construction of structured LDPC codes free of small trapping sets,' IEEE Trans. Inf. Theory, vol. 58, no. 4, pp. 2280-2302, Apr. 2012. 